\title{
Attention and emotion: an ERP analysis of facilitated emotional stimulus processing
}

\author{
Harald T. Schupp, ${ }^{\text {CA }}$ Markus Junghöfer,' Almut I. Weike and Alfons O. Hamm
}

Department of Biological and Clinical Psychology, Institute of Psychology, University of Greifswald, Franz-Mehring-Str. 47, 17487 Greifswald; 'University of Konstanz, Germany

${ }^{\mathrm{CA} C}$ Corresponding Author

Received I3 February 2003; accepted 7 March 2003

\begin{abstract}
Recent event-related potential studies observed an early posterior negativity (EPN) reflecting facilitated processing of emotional images. The present study explored if the facilitated processing of emotional pictures is sustained while subjects perform an explicit non-emotional attention task. EEG was recorded from 129 channels while subjects viewed a rapid continuous stream of images containing emotional pictures as well as task-related checkerboard images. As expected, explicit selective attention to target images
\end{abstract}

elicited large P3 waves. Interestingly, emotional stimuli guided stimulus-driven selective encoding as reflected by augmented EPN amplitudes to emotional stimuli, in particular to stimuli of evolutionary significance (erotic contents, mutilations, and threat). These data demonstrate the selective encoding of emotional stimuli while top-down attentional control was directed towards non-emotional target stimuli. NeuroReport 14:000-000 (c) 2003 Lippincott Williams \& Wilkins.

Key words:

\section{INTRODUCTION}

Environments where resources and dangers are unpredictably distributed in space and time foster the evolution of mechanisms to select significant stimuli for priorized processing. According to this evolutionary perspective, significant emotional stimuli such as food, mating partners, or signals of threat should be particularly effective cues to capture attention [1]. Consistent with this hypothesis, behavioral studies demonstrate the privileged processing of emotionally significant cues. For instance, it was found that fear-related pictures of snakes and spiders were detected faster in grid-pattern arrays of fear-irrelevant pictures (flowers and mushrooms) than vice versa. Furthermore, this effect was more pronounced for subjects with specific fears of these animals [2]. Another line of research demonstrated that schematic threatening faces were detected faster than friendly faces among both neutral and emotional distractors [3]. Additional evidence suggests that this effect is particularly pronounced for highly anxious individuals $[3,4]$.

Attention capture by emotional stimuli has also been observed in patients with spatial neglect, who often remain unaware of stimuli in the contralesional hemifield when presented together with ipsilesional stimuli. Specifically, these patients had increased chances to be aware of stimuli depicting emotional contents (angry or happy faces; spiders) than neutral contents $[5,6]$. Event-related fMRI studies in patients with spatial neglect observed that unseen face stimuli (presented on the contralesional side) engage striate and extrastriate visual structures, including the fusiform gyrus particularly implicated in the processing of faces. Furthermore, the conscious perception of the face stimuli was reflected by increased activity in posterior visual areas and distinct neural structures including frontal, parietal and temporal areas $[7,8]$. In addition, fearful expressions evoked increased activity in limbic structures (amygdala, orbitofrontal cortex) irrespective of whether the fearful faces were perceived [8]. Thus, limbic activation may cause the enhanced perception of emotional stimuli. Conversely, patients with bilateral amygdala damage failed to show the enhanced perception of aversive words in an attentional blink study [9]. These data are consistent with the hypothesis of enhanced perception of emotional stimuli and suggest a role of the amygdala in regulating cortical stimulus processing [10].

The increased sensory processing of emotional compared to neutral stimuli was observed in several fMRI- and PET studies [10,11], including materials such as pleasant or unpleasant pictures [12] and fearful faces [13]. Specifically, increased activations by emotional photographs have been observed in both posterior, visual processing areas (e.g. occipital gyrus) and more anterior, ventral temporal structures (e.g., fusiform gyrus). The selective processing of emotional stimuli in the extended visual cortex was also revealed by electrophysiological studies. Presenting emotional and neutral images from the international affective 
picture series (IAPS), we recently observed that the early posterior negativity (EPN) was the first cortical ERP component reflecting the selective processing of emotional stimuli. The selective EPN component developed around $150 \mathrm{~ms}$ and was maximally pronounced around $260-280 \mathrm{~ms}$ after picture onset. Furthermore, the amplitude of the EPN was most pronounced for stimuli of high evolutionary significance, i.e. erotic images and pictures of mutilations $[14,15]$. Interestingly, the similarity of the selective processing of emotional cues and explicitly attended stimuli has been noted in fMRI and ERP studies [11,15]. For instance, fMRI studies reveal the selective processing of attended stimuli in multiple extrastriate visual structures, possibly extending to very early processing areas $[11,16]$. ERP studies also observed the selective processing of attended cues within the visual cortex [16,17]. In particular, paying attention to specific stimulus characteristics (such as color or form) elicits a temporo-occipital selection negativity in a time window lasting from 150 to $350 \mathrm{~ms}$ [17].

A recent fMRI study compared effects of attention and emotion more directly [18]. Presenting fearful and neutral faces at task-relevant or task-irrelevant locations, amygdala and right-fusiform gyrus activation was observed independent of the attentional focus. Similarly, the present study used an electrophysiological approach to study the hypothesis of obligatory selective emotional processing while subjects pursue an explicit non-emotional attention task. Towards this end, (task-irrelevant) pleasant, neutral, and unpleasant images were presented while subjects had to detect target checkerboard images interspersed in the picture sequence. In order to implement a high perceptual load [19], stimuli were presented as a rapid continuous stream with individual presentation times of $333 \mathrm{~ms}$. Our findings reveal increased EPN amplitudes to pleasant and unpleasant stimuli, particularly pronounced for stimuli of high evolutionary significance, while behavioral and electrophysiological responses to the task stimuli demonstrate successful top-down attentional control to non-emotional stimuli.

\section{MATERIALS AND METHODS}

Participants: Participants were 15 (eight females, 14 righthanded) introductory psychology students from the University of Greifswald. They received course credits toward their research requirements.

Experimental stimuli and task: Pleasant, neutral, and unpleasant pictures $(n=700)$ from the IAPS series were presented [1]. The three categories differed significantly from each other in their normative valence ratings (mean $=6.8,5.1$ and 2.6 for pleasant, neutral, and unpleasant contents on a 1-9 scale). Mean arousal levels for both emotional categories were significantly higher than for neutral contents (5.7, 3.8 and 6.1 for pleasant, neutral, and unpleasant contents, respectively). Task-related stimuli were four distinct checkerboard images. Red/white or yellow/ white checkerboard images contained either a single black or white rectangle centered in the middle of the image. The subjects were asked to count the images with the black or white center rectangle (35 presentations each), respectively.
Affective and task-related images were shown in a perceptually random sequence determined in two steps. First, separate random series of the IAPS pictures and checkerboard images $(n=70)$ were constructed. Second, the checkerboard sequence was interspersed in the IAPS picture sequence with a mean lag of 10 pictures in between (range 2-18). Pictures were presented as a continuous stream of images on a 21 inch EIZO F77 computer screen located $\sim 100 \mathrm{~cm}$ in front of the participant, without perceivable interstimulus intervals ( $85 \mathrm{~Hz}$ refresh rate). Each individual picture was displayed for $333 \mathrm{~ms}$.

Procedure: Participants read and signed an informed consent form and the dense sensor electrode cap was attached. In order to minimize effects due to stimulus novelty, the participants were familiarized with the complete picture stimulus set. Subjects were instructed to count the number of checkerboard images with a black (or white) rectangle in the center. The subject room was dimly lit during video presentation.

Data collection, reduction, and analysis: Brain and ocular scalp potential fields were measured with a 129 lead geodesic sensor net, on-line bandpass filtered from 0.01. to $100 \mathrm{~Hz}$, and sampled at $250 \mathrm{~Hz}$ using Netstation software and EGI amplifiers. Electrode impedance was kept below $30 \mathrm{k} \Omega$, which is appropriate for this type of electroencephalogram (EEG) amplifier. Data were recorded continuously with the vertex sensor as reference electrode. A $30 \mathrm{~Hz}$ digital low pass filter was applied off-line to the continuous EEG data. Stimulus synchronized epochs lasting from $100 \mathrm{~ms}$ before until $800 \mathrm{~ms}$ after picture onset were extracted. Data editing and artifact rejection were based on an elaborate method for statistical control of artifacts, specifically tailored for the analyses of dense sensor ERP recordings [20]. Data reported are based on an average reference.

Explicit attention task: Explicit attentional orienting to target checkerboard images was expected to elicit enlarged parietal P3 amplitudes. Thus, separate average waveforms were calculated for target and non-target checkerboard stimuli for each sensor and participant. For statistical analyses, P3 responses to the checkerboard images were scored as an area measure in the time interval of 468-532 ms after stimulus onset for left and right parietal sensor clusters and subjected to repeated measures ANOVA including the factors stimulus (target $v$ s non-target) and hemisphere (left vs right).

Selective emotion processing: In order to extract the EPN from the EEG signal, separate average waveforms for pleasant, neutral, and unpleasant pictures were calculated for each sensor and participant. Since the P3 component to the task-related checkerboard images occurred at a time at which the following picture has already been displayed, these trials were excluded from analyses. Thus, contamination of the ERP waveforms to the IAPS pictures by $\mathrm{P} 3$ waves to the preceding task-related checkerboards was circumvented. For statistical analyses [14,15], the EPN was scored as mean amplitude of left and right temporo-occipital sensor clusters, each comprising seven sensor sites (the six sensors 
surrounding sensors 64 (left) and 96 (right), respectively). Three consecutive time windows were analyzed (P1: 88-152, N1: 160-224, and N2: 232-292 ms). EPN amplitude was subjected to ANOVA analyses including the factors affect (pleasant, neutral, unpleasant), laterality (left, right), and time (P1, N1, and N2). Further analyses tested the hypothesis that the EPN amplitude is particularly augmented for pictures of high evolutionary significance. Thus, for pleasant valence, EPN amplitudes to erotic materials were compared to pictures of less evolutionary significance (e.g., sports, family scenes). Similarly, for negative valence, pictures of mutilations and threat were compared to pictures of less evolutionary significance (e.g., contamination, grief, accidents).

For effects involving repeated measures, the Wilks Lambda procedure was used to correct for violations of sphericity.

\section{RESULTS}

Explicit attention task: P3 component and behavioral performance: Behavioral and ERP measures served to assure that subjects successfully performed the explicit task. Behavioral performance in the target discrimination task was high. Nine subjects counted the checkerboard targets entirely correct, the remaining subjects miscounted one or two of the targets. Event related potentials also demonstrated that subjects paid attention to the explicit task. As illustrated in Fig. 1 for a representative left (54) and right (80) parietal sensor, task-related checkerboard images were associated with P3 waves, particularly pronounced for the target stimuli $(6.3 \mu \mathrm{V})$ compared to non-target checkerboards $(2.9 \mu \mathrm{V} ; \mathrm{F}(1,14)=22.2, p<0.0001)$.

Selective emotion processing: Although subjects performed an explicit non-emotional attention task, emotional stimuli were nonetheless selectively processed as shown by

P3 Amplitudes to Checkerboard Stimuli

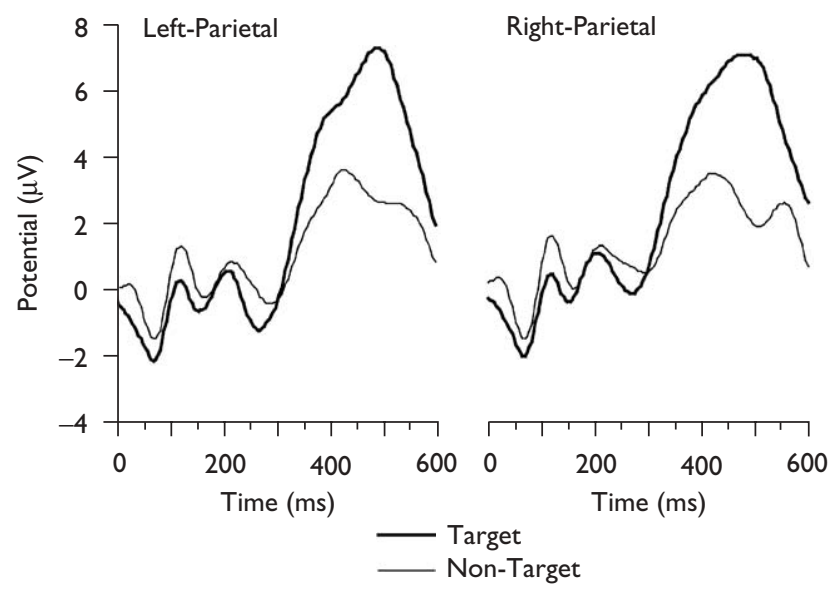

Fig. I. Grand-averaged ERP responses at left (54) and right (80) parietal sensors to target and non-target checkerboard stimuli. P3 waves were exclusively observed for checkerboard images, and most pronounced for target images.

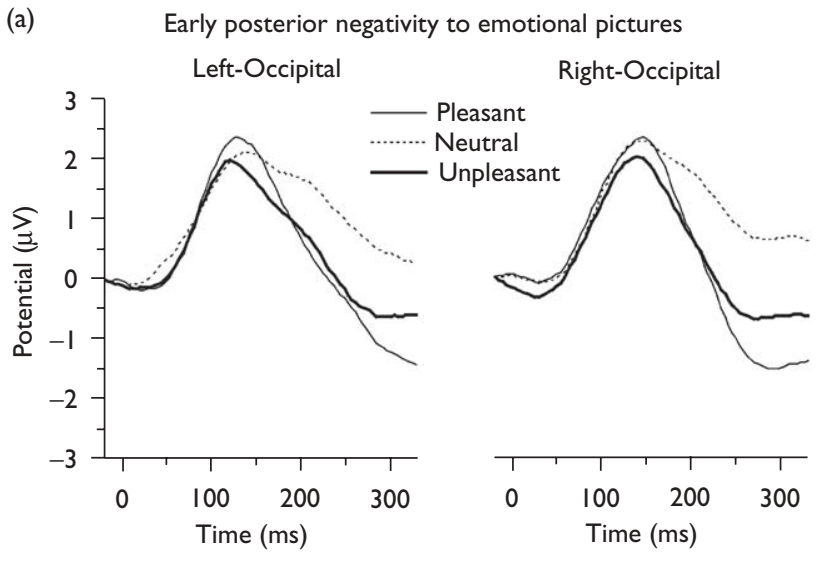

(b)

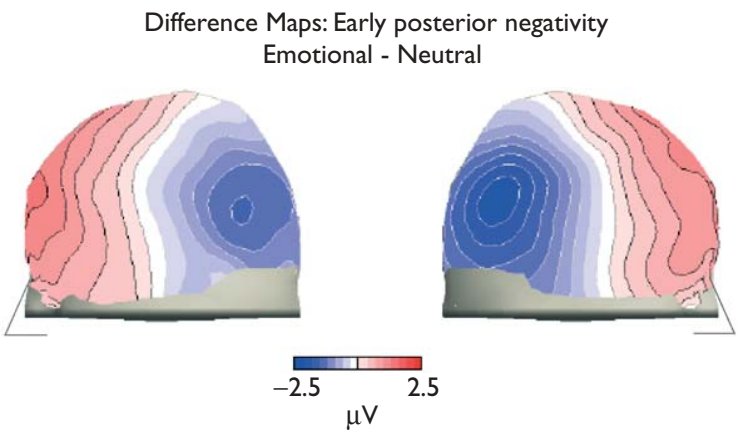

Fig. 2. (a) Grand-averaged ERP waveforms to pleasant, neutral, and unpleasant images. A representative left (64) and right (96) hemispheric occipital sensor was selected for representing enlarged EPN amplitudes elicited by pleasant and unpleasant images. (b) Scalp potential maps reveal the topography of the emotional posterior negativity for the time window (232-296 ms) of strongest affect discrimination ([(Pleasant-Neutral) + (Unpleasant-Neutral)]/2). Contour lines were spaced every $0.25 \mu \mathrm{V}$. Illustrated is a left- and right-side view of the model head.

increased EPN amplitudes to pleasant and unpleasant compared to neutral pictures (affect: $\mathrm{F}(2,13)=11.2$, $p<0.001)$. As illustrated in Fig. 2a, the selective encoding of emotional pictures was not reliably observed for the P1 time window, but developed around $150 \mathrm{~ms}$ as captured by the N1 window and was most pronounced in the N2 window (affect $\times$ time: $\mathrm{F}(4,11)=12.3, p<0.0001$ ).

Separate analyses of the N1 time window revealed that pleasant and unpleasant pictures elicited significantly greater temporo-occipital negativity than did neutral images $(\mathrm{F}(1,14)=11.4$ and 16.6, $p<0.001$; affect: $\mathrm{F}(2,13)=9.5$, $p<0.01)$. Augmented EPN amplitudes to pleasant and unpleasant pictures were even more pronounced in the N2 window $\quad(\mathrm{F}(1,14)=72.4$ and 26.8, $p<0.0001$, affect: $\mathrm{F}(2,13)=37.0, p<0.0001)$. The comparison between pleasant and unpleasant images was not significant in either the $\mathrm{N} 1$ or $\mathrm{N} 2$ time window $(\mathrm{F}(1,14)<2.8, p>0.1)$. Effects of laterality were indicated for the $\mathrm{N} 2$ peak by a marginally significant interaction of affect $\times$ laterality $(\mathrm{F}(2,13)=3.1$, $p=0.08)$, indicating that affective modulation was particu- 
larly pronounced over right temporo-occipital sensor sites (Fig. 2b).

Further analyses of the N2 amplitude explored the hypothesis that enlarged EPN amplitudes are particularly pronounced for stimuli of high evolutionary significance. Exploring pleasant images, pictures depicting erotic materials were associated with enlarged EPN amplitude over temporo-occipital sensors compared to other, evolutionary less relevant pleasant picture contents $(F(1,14)=45.6$, $p<0.001)$. Similarly, pictures of mutilations and threat elicited greater EPN amplitudes than did evolutionarily less relevant unpleasant pictures $(\mathrm{F}(1,14)=25.8, p<0.001)$.

\section{DISCUSSION}

An evolutionary perspective suggests that emotional cues are particularly effective to capture attention, possibly reflecting a default setting irrespective of explicit attentional orienting [1]. Consistent with the hypothesis of privileged processing of emotional cues, the present study observed the selective processing of emotional cues as reflected by increased EPN amplitudes, in particular to stimuli of high evolutionary significance, i.e. erotic displays, pictures of mutilations or threat. Notably, the selective processing of emotional cues was observed while the subject performed an explicit non-emotional attention task. In addition, the rapid and continuous rate of picture presentation assured a high perceptual load. These findings are complemented by research demonstrating enlarged EPN amplitudes to emotional faces, in particular expressions of fear and threat $[21,22]$. Both studies observed selective emotional processing while the subjects' explicit attention was directed towards non-emotional features of the emotional and neutral stimuli (gender or facial orientation). The present study extended these findings by demonstrating selective emotion processing when the explicit attention task did not require the categorization of the emotional or neutral stimuli. Taken together, the data are consistent with the notion of an early tagging of emotional stimuli at the stage of sensory stimulus encoding.

The effects of emotional significance in the posterior negativity in our data (Fig. 2) might be secondary to appraisal of significance in the amygdala. Activation of the amygdala has been observed for aversive pictures [23], fearful faces [13], and recently also for erotic images [23]. Furthermore, amygdala activation does not depend on conscious identification of the stimuli since increased cerebral blood flow in the right amygdala has been observed to masked presentations of threatening faces [24]. Such unconscious activation of the amygdala appears to be occasioned through subcortical visual pathways, according to imaging data from a patient with visual blind sight [25]. Furthermore, the amygdala has multiple pathways that might regulate the allocation of processing resources for further analysis of emotional stimuli at the cortical level. Thus, the amygdala might facilitate selective processing in the visual cortex by direct projections, connections to anterior attention networks or via ascending neuromodulatory systems $[10,11]$.

The present findings are also in line with fMRI studies demonstrating the selective activation of the amygdala and fusiform gyrus to fearful faces independent of whether the faces were presented at attended or unattended spatial locations [18]. However, a recent study, employing a demanding spatial attention task, failed to observe amygdala and fusiform activation when attention was diverted from face processing [26]. The obligatory selective processing of emotion might therefore depend on the availability of processing resources. Future studies are needed to further probe the competition for selective processing as a function of emotional intensity and explicit attention demands.

\section{CONCLUSION}

In a world where various stimuli compete for attentional resources, the fast and reliable detection of hostile and hospitable cues is considered to facilitate adaptive behavior finally promoting survival and reproductive success. An early, automatic tagging of emotional stimuli might assist the selection of significant stimuli for priorized processing. Consistent with this hypothesis, the sensory-driven selective processing of emotional pictures was observed in the context of a primary task involving explicit (top-down) attentional orienting. Research underway determines the generality of these findings and explores the selective affective processing in cross-modal designs and various levels of task demands.

\section{REFERENCES}

1. Lang PJ, Bradley MM and Cuthbert BN. Motivated attention, Affect, activation, and action. In: Lang PJ, Simons RF and Balaban M, eds. Attention and Orienting, Sensory and Motivational Processes. Mahwah, NJ: Lawrence Erlbaum Associates; 1997, pp. 97-135.

2. Öhman A, Flykt A and Esteves F. J Exp Psychol Gen 130, 466-478 (2001).

3. Öhman A, Lundqvist D and Esteves F. J Pers Soc Psychol 80, 381-396 (2001)

4. Mogg K, Millar N and Bradley BP. J Abnorm Psychol 109, 695-704 (2000).

5. Vuilleumier P and Schwartz S. Neuroreport 12, 1119-1122 (2001).

6. Vuilleumier P and Schwartz S. Neurology 56, 153-158 (2001).

7. Driver J, Vuilleumier P, Eimer M and Rees G. Neuroimage 14, S68-S75 (2001).

8. Vuilleumier P, Armony JL, Clarke K et al. Neuropsychologia 40, 2156-2166 (2002).

9. Anderson AK and Phelps EA. Nature 411, 305-309 (2001).

10. Davis M and Whalen PJ. Mol Psychiatry 13-34 (2001).

11. Pessoa L, Kastner S and Ungerleider LG. Cogn Brain Res 15, 31-45 (2002).

12. Lang PJ, Bradley MM, Fitzsimmons JR et al. Psychophysiology 35, 199-210 (1998).

13. Morris JS, Friston KJ, Buchel C et al. Brain 121, 47-57 (1998).

14. Junghöfer M, Bradley MM, Elbert TR et al. Psychophysiology 38, 175-178 (2001).

15. Schupp HT, Junghöfer M, Weike AI and Hamm AO. Psychol Sci 14, 7-13 (2003)

16. Noesselt T, Hillyard SA, Woldorff MG et al. Neuron 35, 575-587 (2002).

17. Näätänen R. Attention and Brain Function. Hillsdale, NJ: Lawrence Erlbaum Associates; 1992

18. Vuilleumier P, Armony JL, Driver J and Dolan RJ. Neuron 30, 829-841 (2001)

19. Luck SJ, Woodman GF and Vogel EK. Trends Cogn Sci 4, 432-440 (2000).

20. Junghöfer M, Elbert T, Tucker D and Rockstroh B. Psychophysiology 37, 523-532 (2000).

21. Sato W, Kochiyama T, Yoshikawa S and Matsumura M. NeuroReport 12, 709-714 (2001).

22. Schupp HT, Stockburger J, Bahlmann J et al. Psychophysiology 38, S87 (2001).

23. Hamann SB, Ely TD, Hoffman JM and Kilts CD. Psychol Sci 13, 135-141 (2002).

24. Whalen PJ, Rauch SL, Etcoff NL et al. J Neurosci 18, 411-418 (1998) 
25. Morris JS, DeGelder B, Weiskrantz L and Dolan RJ. Brain 124, 1241-1252 (2001).
26. Pessoa L, McKenna M, Gutierrez E and Ungerleider LG. Proc Natl Acad Sci USA 99, 11458-11463 (2002).

Acknowledgements: This work was supported by the German Research Foundation (DFG) Grants Schu I074/7-I and Hamm I563/ 10-2. We are grateful to the help of Jessica Stockburger, Silva Rosolski, and Andrea Sigmund. 